\title{
A comparison of body composition measurement techniques
}

\author{
L. W. Beck, A. Petropoulou, S. Hillier and M. E. Clegg \\ Functional Food Centre, Department of Sport and Health Sciences, Oxford Brookes University, Gipsy Lane, \\ Oxford, OX3 OBP, UK
}

\begin{abstract}
An understanding of the reproducibility of body composition measurements is essential to ascertain the effectiveness of a weight loss intervention. Air displacement plethysmography (ADP) and bioelectrical impedance (BIA) are two easy-to-use measures of body composition. Previous studies have not compared the reproducibility of both methods. The aim of this study was to assess the reproducibility of ADP and BIA and compare them to eachother and skinfold measurements.

Forty-one healthy participants (22 female, 19 male; age: 23.0 (SD 3.1) yr; height: 1.74 (SD 0.10) m; weight: 67.4 (SD 9.9) kg) were tested on two occasions following an overnight fast. On their first test day, participants' height, weight and \% body fat (BF) were measured using ADP (BODPOD, COSMED, Rome, Italy) and BIA (Tanita BC-418 MA, Yiewsley, UK). Measurements were replicated to assess the within-day reproducibility of both ADP and BIA. On their second test day, participants were again tested using ADP and BIA as well as having skinfold measurements taken. Comparisons of within- and between-day reproducibility and comparisons between measurement techniques were completed using Pearson correlations and Bland-Altman analysis.

The Pearson correlation showed good within-day and between-day relationships for ADP and BIA measurements of \% BF. It also demonstrated that the two methods had a high correlation between them. Bland-Altman analysis indicated agreement within-day and between-day for ADP and BIA measurements of \% BF. For ADP, the within-day mean difference was 0.3 (SD 1.7 ) $\%$ and between-day was 0.1 (SD 2.1) \%. For BIA, the differences were 0.1 (SD 0.4) \% within-day and 0.5 (SD 1.8) \% between-day. The mean difference between BIA and ADP was 3.1 (SD 4.1) \%. From the skinfold data, the best agreement with ADP had a mean difference of 0.3 (SD 2.8) $\%^{(2,3)}$ compared to 5.4 (SD 3.6) $\%^{(1)}$ and 6.1 (SD 3.5) $\%^{(4)}$. The BIA had mean differences with the skinfold measurements of 1.9 $(\mathrm{SD} 4.2) \%^{(1)}, 3.8(\mathrm{SD} 4.0) \%^{(2,3)}$ and $2.7(\mathrm{SD} 3.8) \%^{(4)}$.
\end{abstract}

\begin{tabular}{lcc}
\hline & Within day & Between day \\
\hline ADP & $0.977 ; P<0.001$ & $0.965 ; P<0.001$ \\
BIA & $0.999 ; P<0.001$ & $0.977 ; P<0.001$ \\
\hline
\end{tabular}

Within-day and between-day correlations ( $\mathrm{R} ; \mathrm{P}$ value) of $\%$ body fat from ADP and BIA

\begin{tabular}{lcllll}
\hline & ADP & \multicolumn{1}{c}{ BIA } & \multicolumn{1}{c}{ Skinfold $^{(1)}$} & \multicolumn{1}{c}{ Skinfold $^{(2,3)}$} & Skinfold $^{(4)}$ \\
\hline ADP & X & $0.886 ; P<0.001$ & $0.899 ; P<0.001$ & $0.930 ; P<0.001$ & $0.908 ; P<0.001$ \\
BIA & X & X & $0.902 ; P<0.001$ & $0.902 ; P<0.001$ & $0.918 ; P<0.001$ \\
Skinfold $\left.^{1}\right)$ & X & X & X & $0.972 ; P<0.001$ & $0.960 ; P<0.001$ \\
Skinfold $\left.^{2,3}\right)$ & X & X & X & X & $0.972 ; P<0.001$ \\
\hline
\end{tabular}

Correlations (R; $P$ value) between ADP, BIA and skinfold measurements of \% body fat.

The current data indicates that both ADP and BIA have good within- and between-day agreement however the two measurements demonstrated a mean difference of $3.1 \%$ BF between them. The data indicates that ADP and BIA cannot be used interchangeably.

1. Durnin JVGA \& Wormersley J (1974) Br J Nutr 32, 77-97.

2. Jackson AS \& Pollock ML (1978) Br J Nutr 40, 497-502.

3. Jackson AS, Pollock ML \& Ward A (1980) Med Sci Sports Exerc 12, 175-82.

4. Peterson MJ, Czerwinski SA \& Siervogel RM (2003) Am J Clin Nutr 77, 1186-91. 